\title{
Ulrike Woggon
}

\section{Optical Properties of Semiconductor Quantum Dots}

With 126 Figures 


\section{Contents}

1. Introduction $\ldots \ldots \ldots \ldots \ldots \ldots \ldots \ldots \ldots \ldots \ldots \ldots \ldots \ldots \ldots, 1$

2. Growth of Nanocrystals ...................... 7

2.1 Growth of Nanocrystals in Glass Matrices ............ 8

2.1.1 The Diffusion-Controlled Growth Process.......... 8

2.1.2 Preparation of II-VI Nanocrystals ............. 13

2.1.3 Other Semiconductor Materials in Glass Matrices .... 24

2.2 Growth of Nanocrystals in Organic

and Related Matrices ...................... 26

2.2.1 Chemical Preparation Methods.............. 26

2.2.2 Size-Selection Techniques ................... 29

2.2.3 Sandwiches and Quantum Dot Quantum Wells ...... 30

2.3 Structural Data .............................. 33

2.4 Influence of Interfaces . . . . . . . . . . . . . . . . . . . 36

2.5 Epitaxial Growth $\ldots \ldots \ldots \ldots \ldots \ldots \ldots \ldots \ldots \ldots \ldots \ldots \ldots \ldots \ldots$

3. Energy States $\ldots \ldots \ldots \ldots \ldots \ldots \ldots \ldots \ldots \ldots \ldots \ldots \ldots, 43$

3.1 One-Electron-Hole-Pair States . .................. 43

3.1.1 The Particle-in-the-Box Model ............... 43

3.1.2 Coulomb Interaction . . . . . . . . . . . . . . . . . 48

3.1.3 Mixing of Hole States . . . . . . . . . . . . . . . . 52

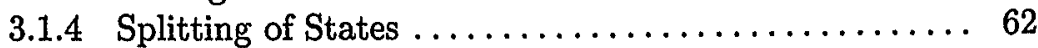

3.1.5 Indirect-type Quantum Dots ................ 65

3.1.6 Experiments to Identify One-Pair States .......... 68

3.2 Two-Electron-Hole-Pair States . . . . . . . . . . . . . . . . 80

3.2 .1 Theory ............................. 80

3.2.2 Experiments to Identify Two-Pair States ......... 83

3.2.3 Optical Gain........................... 91

3.3 Many Particle Interaction . . . . . . . . . . . . . . 97

4. Dielectric Effects $\ldots \ldots \ldots \ldots \ldots \ldots \ldots \ldots \ldots \ldots \ldots \ldots \ldots \ldots$

4.1 Optical Properties of Composites ................. 103

4.2 Surface Polarization and Charge Separation........... 110 


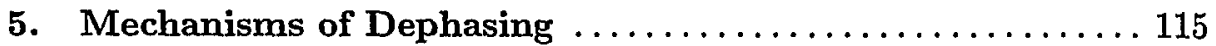

5.1 Coupling of Electron-Hole Pairs with Phonons .......... 116

5.1.1 Phonons in Quantum Dots

and Coupling Mechanisms ................... 116

5.1.2 Raman Scattering ...................... 124

5.1.3 Photoluminescence

and Photoluminescence Excitation Spectroscopy ..... . 129

5.1.4 Hole-Burning and Saturation Spectroscopy ........ 135

5.1.5 Four-Wave Mixing .......................... 137

5.2 Energy Relaxation ............................ 142

5.3 Scattering at Defects and Interfaces . . . . . . . . . . . . 149

5.4 Carrier-Carrier Scattering ..................... 151

6. Trap Processes . ........................... 159

6.1 Localization, Trapping and Transfer $\ldots \ldots \ldots \ldots \ldots \ldots \ldots$

6.2 Kinetic Models ............................... 167

6.3 Trap Processes and Nonlinear Optical Properties ......... 174

7. Effects of Static External Fields ................. 179

7.1 Electric Field Effects . . . . . . . . . . . . . . . . . . . 179

7.2 Magnetic Field Effects ....................... 187

7.3 External Fields Acting as Confining Potentials ........... 193

7.3.1 Magnetic Field Confined Electrons . ............ 193

7.3.2 Electric Field Confined Electrons

and Transport Properties.................. 195

8. Nanocrystals of III-V Compounds ................ 199

8.1 Spherical Quantum Dots in Polymers and Glasses ......... 199

8.2 Quantum Dots Obtained by Deep-Etching

and Interdiffusion ........................... 200

8.3 Quantum Dots due to Spatially Isolated

Potential Fluctuations .......................... 201

8.4 Quantum Dots Resulting from

Self-organized Epitaxial Growth ...................... 205

8.5 Stressor-Induced Quantum Dots .................. 208

9. Nanocrystals of Indirect-Gap Materials .............. 209

9.1 Theoretical Description ....................... 209

9.2 Silicon Nanocrystals

and Quantum Structures in Porous Silicon ............. 212

9.2.1 Preparation Methods ........................ 213

9.2.2 Luminescence and Luminescence Dynamics ......... 214

9.2.3 Polarization of Luminescence . . . . . . . . . . . . . 218

10. Concepts of Applications $\ldots \ldots \ldots \ldots \ldots \ldots \ldots \ldots \ldots \ldots, 223$

References ...................................... 231

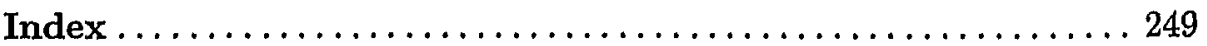

